\section{Clinical Memoranda}

\section{Carcinoma of Bronchus Simulating a Mild Septicaemia}

The case reported below illustrates the difficulty in arriving at a correct conclusion in some cases of pyrexia. There have been several similar cases in this hospital, where carcinoma of the lung with prolonged pyrexia has led to a difficulty in diagnosis. It is also of interest on account of the absence of symptoms, in spite of marked involvement of the suprarenals.

An engineer aged 27 years, a patient of Dr. Rowlands of Waterloo, was admitted to Walton Hospital in June, 1935. Dr. Rowlands kindly furnished the following notes:

"This patient has been in another hospital for some months. No definite diagnosis was made, except that it was suggested that it might be a mild streptococcal septicaemia.

" About a year ago he started to have pains in his back, with some frequency of micturition. He still has marked pain, associated with great muscular weakness and mild pyrexia.

"When in hospital the blood was sterile, $x$-ray of the chest, spine, and sinuses proved negative, the Wassermann reaction was negative, and agglutination tests for the enteric group and $B$. abortus were negative. There were no tubercle bacilli in the sputum, and the blood picture showed a secondary anaemia."

On admission to Walton Hospital the patient was bedridden, emaciated, and slightly anaemic. $\mathrm{He}$ ran an irregular temperature, varying between $101^{\circ}$ and $97^{\circ}$, for the whole period, until his death in August, 1935. He complained of pain in the left lumbar region, and the involvement of the pyramidal tracts was manifested by a bilateral extensor response: No definite sensory changes were found, and the cerebro-spinal fluid was normal. The left knee-jerk was absent. A provisional diagnosis of cord tumour was made at this time, and the spine was $x$-rayed, but there was no evidence of any changes suggesting neoplasm. The chest was also $x$-rayed, and the report was: "Bronchopneumonic infiltration of both lungs, probably non-tuberculous." No Bence-Jones protein was found in the urine. The blood count, later, showed a well-marked hypochromic anaemia of the secondary type, with a normal white cell count. Blood culture was negative.

The patient's condition remained fairly constant, but a few days before death he had a series of epileptiform convulsions. There was no pigmentation of his skin throughout.

Post-mortem Report by Dr. Dawbarn

A small primary carcinoma of one of the bronchi was situated about halfway between the hilum and the base of the right lower lobe. Malignant glands were found at the roots of the lungs and many small secondary growths in both lungs. The lungs were congested at the bases. Heart: Very little fat; no valvular disease. Spleen: Ten ounces ; no metastases. Liver: Many metastases up to the size of cherries, and a strawberry gall-bladder. Pancreas, stomach, and intestines : Normal. Kidneys: Several small metastases in each. Right suprarenal: Almost destroyed by growth. Left suprarenal : Extensively involved. Bladder, ureters, prostate, and testes: Normal. Spinal column: Many metastases; several protruding anteriorly from the bodies of the lower lumbar vertebrae, and several protruding into the spinal canal in the lumbar and dorsal regions, pressing on the cord. Brain : ? one metastasis over the upper part of the right fissure of Rolando ; otherwise normal.

Microscopy : Sections show a carcinoma of the bronchus with metastases in the liver and suprarenal, but not in the meninges or brain. The growth is of the adeno-columnar cell type. This is typical of growths from small bronchi, according to Geschickter. ${ }^{1}$

I wish to thank the medical superintendent, Dr. McWilliam, and his assistants for their help.

Liverpool.

T. W. WADSWORTh, M.D., F.R.C.P.

\section{Reviews}

\section{THE EXTRA PHARMACOPOEIA: PART II}

We welcome the appearance of Part II of the twentieth edition of Martindale and Westcott's Extra Pharmacopoeia. ${ }^{1}$ This work has maintained its popularity for generations, and has come to be regarded as an almost indispensable source of quick information.

When Mr. W. H. Martindale died in 1933 there was a general anxiety as to the future of these useful volumes, but we are glad to say that the Pharmaceutical Society has undertaken the responsibility of preparing future editions. Mr. Martindale performed a great service to the medical profession by taking charge of this work for many years, and those who used the volumes marvelled equally at his amazing energy and his breadth of knowledge. The work was, however, rapidly outgrowing the powers of any one individual, and, in fact, Mr. Martindale's devotion to this task undoubtedly hastened his death. The Pharmaceutical Society has arranged for the volumes to be edited by Mr. C. E. Corfield, the editor of the British Pharmaceutical Codex, with the assistance of a number of experts. It is proposed to maintain the general character of the volumes, but to make a thorough revision of the kind that is bound to be required periodically in the case of works that gradually expand throughout frequent editions.

The contents of the volume under review fall into four chief sections. First, analytical addenda to chemicals and materia medica described in vol. i (204 pages); secondly, data on chemistry and chemical pathology (156 pages); thirdly, notes on dietetics (146 pages); fourthly, bacteriological and clinical notes (236 pages). In the analytical section particular attention has been paid to drugs for which new standards have been introduced, and these notes incorporate the advances made in the current editions of the B.P. and the B.P.C. Information is also given about the standards provided by foreign pharmacopoeias. The section on dietetics is particularly interesting, and includes an excellent summary of the present state of knowledge of the chemistry of vitamins. Forty pages are devoted to the chemistry of milk, and an abstract is given of the current official regulations and tests. The bacteriological and clinical notes contain a large amount of varied information. The sections on sterilization, disinfection, and-chemotherapy summarize clearly the principles involved in these processes. No fewer than twenty-five pages are devoted to $x$ rays, radium, and electrotherapy.

In general, we may say that the volume maintains its reputation for presenting a great deal of varied information in a compact form. It has been compiled by a team of specialists, including not only pharmacists and organic chemists, but also experts on such subjects as biological standardization, vitamins, and radiology. Consequently the level of accuracy is very high, and the articles provide excellent summaries of recent knowledge in a wide range of sciences.

\section{PSYCHIATRY}

Dr. MacFie CAMPBell has written a useful book entitled Destiny and Disease in Mental Disorders. ${ }^{2}$ The contributions of this writer are well known to psychiatrists in this country, and they are always stimulating and encouraging. Those who have spent many years in the care of mental patients cannot have failed to come to the conclusion that

${ }^{1}$ The Extra Pharmacopoeia, Part II. Twentieth edition. The Pharmaceutical Press and $\mathrm{H}$. K. Lewis and Co., Ltd., London. 1935. (Pp. 889. 22s. 6d. net.)

${ }^{2}$ London: Chapman and Hall, Ltd. 1935. (Pp. 207. 10s. 6d. net.) 